\title{
THE EVEN CYCLE PROBLEM FOR DIRECTED GRAPHS
}

\author{
CARSTEN THOMASSEN
}

\section{INTRODUCTION}

The problem of deciding if a given digraph (directed graph) has an even length dicycle (i.e., directed cycle of even length) has come up in various connection. It is a well-known hard problem to decide if a hypergraph is bipartite. Seymour [11] (see also [15]) showed that a minimally nonbipartite hypergraph has at least as many hyperedges as vertices. He characterized those with the same number of hyperedges and vertices in terms of digraphs with no even length dicycle.

Problems in qualitative linear algebra have motivated the concept of a signnonsingular matrix. This is a real matrix $A$ such that each matrix $A^{\prime}$ with the same sign pattern as $A$ (i.e., corresponding entries in $A$ and $A^{\prime}$ either have the same sign or both equal 0 ) has linearly independent columns. Klee et al [4] showed that it is hard to decide if a given matrix is sign-nonsingular. However, they left an important special case open: They showed that the problem of deciding if a square matrix is sign-nonsingular is equivalent with the even length dicycle problem for digraphs.

Although the concepts of the determinant $\operatorname{det} A$ and the permanant $\operatorname{per} A$ of a real square matrix $A$ are analogous, they are not equally easy to compute. Computing per $A$ is hard even for the $0-1$ case (see [17]). That special case amounts to finding the number of perfect matchings in bipartite graphs, a problem that plays a role in models in physics [3] and chemistry [10]. Polya [8] suggested that one might try to multiply some entries in a matrix $A$ by -1 and thereby obtain a matrix $A^{\prime}$ such that $\operatorname{per} A=\operatorname{det} A^{\prime}$. Vazirani and Yannakakis [17] showed that the problem of finding such a matrix $A^{\prime}$ is equivalent to the even length dicycle problem.

In 1975 Lovász [6] raised two fundamental questions on the even length dicycle problem.

L(1) Does there exist a natural number $k$ such that any digraph in which there are at least $k$ arcs leaving each vertex has an even length dicycle?

$\mathrm{L}(2)$ Does there exist a natural number $k$ such that any strongly $k$-connected digraph has an even length dicycle? ("Strongly $k$-connected" means that the removal of any vertex set of cardinality $<k$ leaves a digraph in which each vertex can be reached by a directed path from each other vertex.)

Received by the editors January 30, 1990.

1991 Mathematics Subject Classification. Primary 05C20, 05C38. 
For undirected graphs there is a host of results on configurations that are guaranteed by large degrees or large connectivity. For a survey, see [15]. Moreover, questions involving large connectivity or large minimum degree are equivalent in the undirected case since large connectivity implies large minimum degree and large minimum degree implies, by a result of $W$. Mader, the presence of a subgraph of large connectivity (see [15]). This changes dramatically when we go to digraphs: $\mathrm{L}(1)$ was answered in the negative by the present author [13], and the present paper provides an affirmative answer to $L(2)$. The best constant in $L(2)$ is 3 . Interesting partial results on $L(1), L(2)$ were found by Friedland [2] who used (the validity of) the van der Waerden conjecture to prove the following: If $m$ is a natural number, $m \geq 7$, and $D$ is a digraph in which there are precisely $m$ arcs leaving and entering each vertex, then $D$ has an even length dicycle. A related result with an equally interesting proof, based on the so-called Lovász Local Lemma, was obtained by Alon and Lineal [1]. Friedland conjectured that his result also holds for $m \geq 3$. A stronger result was suggested by the author [13, Question 1]. Both conjectures follow from the result of the present paper. Vazirani and Yannakakis [17] suggested that one might obtain an affirmative answer to $\mathrm{L}(2)$ from [2] by proving that, for $r$ sufficiently large, every strongly $r$-connected digraph contains a digraph satisfying the assumption of [2]. We show in this paper that no such $r$ exists.

\section{TERMINOLOGY AND PRELIMINARIES}

A digraph $D$ consists of a finite set $V(D)$ of vertices and a set $E(D)$ of ordered pairs $x y$ of distinct vertices called arcs. If the arc $e=x y$ is present, we say that $x$ dominates $y$ and that $e$ leaves $x$ and enters $y$. More generally, if $A$ and $B$ are disjoint vertex sets such that $x \in A, y \in B$, then $e$ leaves $A$ and enters $B$. The number of arcs leaving $x$ is the outdegree of $x$ and is denoted $d^{+}(x, D)$. The indegree $d^{-}(x, D)$ is defined analogously.

A dipath is a digraph with distinct vertices $x_{1}, x_{2}, \ldots, x_{m}$ and $\operatorname{arcs} x_{1} x_{i+1}$, $i=1,2, \ldots, m-1$. We call this an $x_{1}-x_{m}$ dipath. More generally, if $A$ and $B$ are disjoint vertex sets in a digraph $D$, then an $A-B$ dipath is an $x-y$ dipath $P$ such that $x \in A, y \in B$ and $V(P) \cap(A \cup B)=\{x, y\}$. We say that $y$ can be reached from $x$ in $D$ if such a $P$ exists. If each vertex of $D$ can be reached from each other vertex of $D$, then $D$ is strong. If $A \subseteq V(D) \cup E(D)$, then $D-A$ is obtained from $D$ by deleting $A$ and all arcs leaving or entering vertices in $A$. We write $D-x$ instead of $D-\{x\}$ if $x \in V(D) \cup E(D)$. If $A \subseteq V(D)$, then the subdigraph $D(A)$ induced by $A$ is defined as $D-(V(D) \backslash A) . D$ is strongly $k$-connected if $|V(D)| \geq k+1$ and $D-A$ is strong for each vertex set $A$ of cardinality $<k$. A strong component of $D$ is a maximal strong subdigraph. An initial component (respectively terminal component) of $D$ is a component $H$ of $D$ such that no $\operatorname{arcs}$ of $D$ enters (respectively leaves) $H$. It is easy to see that every digraph has at least one initial component and at least one terminal component.

If $D$ is a digraph, then splitting a vertex $v$ of outdegree or indegree at least 2 in $D$ means that we replace $v$ by two vertices $x$ and $y$ where $x$ dominates $y$. All arcs $v z$ (respectively $u v$ ) in $D$ are replaced by $y z$ (respectively $u x$ ) in the new digraph. Subdividing an arc $x y$ means that we replace $x y$ by an 
$x-y$ dipath that has no intermediate vertices in common with $D$.

A dicycle (more precisely, an $m$-dicycle) is a digraph with vertex set $x_{1}$, $x_{2}, \ldots, x_{m}$ and arc set $x_{1} x_{2}, x_{2} x_{3}, \ldots, x_{m-1} x_{m}, x_{m} x_{1}$. If we add the arcs $x_{1} x_{m}, x_{m} x_{m-1}, \ldots, x_{2} x_{1}$ we obtain a double-cycle (more precisely an $m$ double-cycle). A weak $m$-double-cycle is obtained from an $m$-double-cycle by splitting vertices and subdividing arcs. A weak odd double-cycle is a weak $m$ double cycle for some odd $m$. A digraph $D$ is even if every subdivision of $D$ contains a dicycle of even length. Equivalently, if the arcs of $D$ are assigned weights 0 or 1 , then there is a dicycle of even total weight. A weak odd doublecycle has an odd number of dicycles and every arc is in an even number of dicycles. This implies that every weak odd double-cycle is even. Conversely, we have

Theorem 2.1 [12]. A digraph is even if and only if it contains a weak odd doublecycle.

(For the main result in this paper we only use the trivial part of Theorem 2.1. Only in the last section is Theorem 2.1 used in its full strength.)

Contracting an arc $x y$ of $D$ means that we replace $x, y$ by a single vertex $z$ and all arcs leaving or entering $x$ or $y$ will leave or enter $z$ in the new digraph. (No loops are introduced.)

Lemma 2.2. Let $x y$ be an arc of $D$ such that either $d^{+}(x, D)=1$ or $d^{-}(y, D)$ $=1$. Let $D^{\prime}$ be obtained from $D$ by contracting $x y$ into a vertex $z$. Then $D^{\prime}$ contains a weak $k$-double-cycle if and only if $D$ contains a weak $k$-double cycle.

Proof. Assume that $d^{+}(x, D)=1$. Let $M^{\prime}$ be a weak $k$-double-cycle in $D^{\prime}$. If $M^{\prime}$ contains no arc that in $D$ enters $x$, then $M^{\prime} \subseteq D$. So assume that $M^{\prime}$ contains an arc that enters $x$ in $D$. Let $M$ be the subdigraph in $D$ containing $x, y, x y$, all arcs of $M^{\prime}$, and all vertices of $M^{\prime}$ (except $z$ ). Then $M$ is a weak $k$-double-cycle in $D$. Conversely, the contraction of $x y$ transforms any weak $k$-double-cycle in $D$ into a weak $k$-double-cycle in $D^{\prime}$.

Consider a digraph $D$ that is strong but not strongly 2-connected. Let $v$ be a vertex such that $D-v$ is not strong. Let $H$ be an initial or terminal component of $D$. We define the $H$-reduction of $D$ at $v$ as follows: If $H$ is a terminal component, then the $H$-reduction at $v$ is obtained from $D(V(H) \cup\{v\})$ by adding all the arcs $v z$ for all vertices $z$ in $H$ for which there is an arc in $D$ of the form $u z$ where $u \notin V(H)$. If $H$ is an initial component, then the definition is analogous.

Lemma 2.3. Let $D$ be a strong digraph such that $D-v$ is not strong. Let $H$ be a terminal component of $D-v$. Let $D^{\prime}$ be the $H$-reduction of $D$ at $v$. If $D^{\prime}$ has a weak $k$-double-cycle, then $D$ has a weak $k$-double-cycle.

Proof. Let $M^{\prime}$ be a weak $k$-double-cycle in $D^{\prime}$. If $v z^{\prime}$ is an arc in $M^{\prime}$, then $D$ has an arc $z z^{\prime}$ where $z \notin V(H)$. Let $P$ be a dipath to $z$ from $v$. If there is another arc $v y^{\prime}$ in $M^{\prime}$, then we consider an arc $y y^{\prime}$ (where $y \notin V(H)$ ) and a dipath $P^{\prime}$ to $y$ from $v$. We walk along $P^{\prime}$ backwards from $y$ to $v$ and we stop when we hit $P$. The subdipath of $P^{\prime}$ which we traverse in this way is 
called $P^{\prime \prime}$. Now we replace $v z^{\prime}$ in $M^{\prime}$ by $P$, and, if $v y^{\prime}$ is present in $M^{\prime}$, we replace $v y^{\prime}$ by $P^{\prime \prime}$. This transforms $M^{\prime}$ into a weak $k$-double-cycle in $D$.

Finally we shall use Menger's Theorem (for digraphs), which is presented in almost all books on graph theory.

\section{SUFFICIENT CONDITIONS FOR WEAK 3-DOUBLE-CYCLES}

Lemma 3.1. Let $v$ be a vertex in a strongly 2-connected digraph $D$. If $D-v$ has a dicycle whose vertices all are dominated by $v$ (in $D$ ) or a dicycle whose vertices all dominate $v$, then $D$ contains a weak 3-double-cycle.

Proof. Suppose $C$ is a dicycle whose vertices all dominate $v$. By Menger's Theorem, let $P_{1}, P_{2}$ be two $v-V(C)$ dipaths such that $P_{1} \cap P_{2}=\{v\}$. Then $P_{1} \cup P_{2} \cup C$ union two arcs from $C$ to $v$ form a subdivision of a 3-doublecycle.

Lemma 3.2. Let $v_{1}, v_{2}, v_{3}, v_{4}$ be vertices in a strongly 2-connected digraph $D$ such that $D$ contains the arcs $v_{1} v_{3}, v_{1} v_{4}, v_{2} v_{3}, v_{2} v_{4}, v_{3} v_{4}$. Then $D$ contains a weak 3-double-cycle.

Proof. Let $P_{1}, P_{2}$ be two dipaths from $v_{4}$ to $v_{1}$ and $v_{2}$, respectively, such that $P_{1} \cap P_{2}=\left\{v_{4}\right\}$. If $v_{3} \notin V\left(P_{1}\right) \cup V\left(P_{2}\right)$, then $D-v_{4}$ has a $v_{3}-\left(V\left(P_{1}\right) \cup V\left(P_{2}\right)\right)$ dipath $P_{3}$. Assume without loss of generality that $P_{3}$ intersects $P_{1}$. Now $P_{1} \cup P_{2} \cup P_{3} \cup\left\{v_{1} v_{3}, v_{1} v_{4}, v_{2} v_{3}, v_{3} v_{4}\right\}$ is a weak 3-double-cycle. So we can assume that $v_{3} \in V\left(P_{1}\right)$. Then $v_{3}$ partitions $P_{1}$ into two dipaths $R_{1}$ and $R_{2}$, say. Let $P_{3}$ be a $\left(V\left(R_{1}\right) \cup V\left(P_{2}\right)\right)-V\left(R_{2}\right)$ dipath in $D-v_{3}$.

Then $P_{1} \cup P_{2} \cup P_{3} \cup\left\{v_{1} v_{3}, v_{1} v_{4}, v_{3} v_{4}\right\}$ contains a weak 3-double-cycle.

We are now ready for the main results of this paper.

Theorem 3.3. Let $D$ be a strong digraph such that each vertex has outdegree at least 2. Let $v_{1}, v_{2}, v_{3}$ be vertices such that all other vertices of $D$ have outdegree at least 3. Assume further that, for each vertex $x \neq v_{1}$, every vertex $\neq x$ can be reached from $v_{1}$ in $D-x$. Then $D$ contains a weak 3-double-cycle. In particular, $D$ is even.

Proof. We assume that Theorem 3.3 is false and let $D$ be a counterexample with as few vertices as possible and (subject to that condition) with as few arcs as possible. We shall establish a number of properties of $D$ that will finally result in a contradiction.

(1) $D$ is strongly 2 -connected.

Proof. Suppose $u$ is a vertex such that $D-u$ is not strong. Let $D^{\prime}$ be a terminal component of $D-u$. The assumption of Theorem 3.3 implies that $v_{1} \notin V\left(D^{\prime}\right)$. Let $D^{\prime \prime}$ be the $D^{\prime}$-reduction at $u$. For each vertex $x$ in $D^{\prime}$, $d^{+}\left(x, D^{\prime \prime}\right)=d^{+}(x, D) \geq 2$. Hence $\left|V\left(D^{\prime \prime}\right)\right| \geq 3$. If $d^{+}\left(u, D^{\prime \prime}\right)<2$, then $D^{\prime}$ has a vertex $x$ (namely, the vertex dominated by $u$ in $D^{\prime \prime}$ ) such that $D-x$ has no dipath from $v_{1}$ to $D^{\prime}-x$. This contradiction shows that $d^{+}\left(u, D^{\prime \prime}\right) \geq 2$. Now it is easy to see that $D^{\prime \prime}$ satisfies the assumption of Theorem 3.3 with $u$ playing the role of $v_{1}$. But $D^{\prime \prime}$ contains no weak 3-double-cycle by Lemma 2.3. This contradiction proves (1).

(2) $d^{+}\left(v_{1}\right)=2$. 
Proof. If all vertices of $D$ have outdegree at least 3, then we let $z$ be any vertex dominating $v_{1}$. Then $D-z v_{1}$ satisfies the assumption of Theorem 3.3 with $v_{2}=z$ contrary to the minimality of $D$. So some $v_{i}$ has outdegree 2. As $D$ is strongly 2-connected each $v_{i}(i=1,2,3)$ can play the role of $v_{1}$.

Let $u_{1}$ and $u_{2}$ be the two vertices dominated by $v_{1}$. We show that the notation can be chosen such that the following holds.

(3) If we delete the arc $v_{1} u_{2}$ and contract $v_{1} u_{1}$, then the resulting digraph $D_{1}$ has minimum outdegree at least 2 .

Proof. If (3) is false, then either

(i) $d^{+}\left(u_{1}, D\right)=2$ and $u_{1}$ dominates $v_{1}$ or

(ii) some vertex $z_{1}$ of outdegree 2 (in $D$ ) dominates both $v_{1}$ and $u_{1}$. As we may interchange between $u_{1}$ and $u_{2}$ we can also assume that either

(iii) $d^{+}\left(u_{2}, D\right)=2$ and $u_{2}$ dominates $v_{1}$ or

(iv) some vertex $z_{2}$ of outdegree 2 (in $D$ ) dominates both $v_{1}$ and $u_{2}$. If $z_{1}$ exists (i.e., (ii) holds), then $z_{1} \neq u_{2}$ by Lemma 3.1. Similarly, $z_{2} \neq u_{1}$ if (iv) holds. If both (ii) and (iv) hold, then $z_{1} \neq z_{2}$. If (ii) holds, then (3) holds with the ordered triple $z_{1}, v_{1}, u_{1}$ or $z_{1}, u_{1}, v_{1}$ playing the role of $v_{1}, u_{1}, u_{2}$. (To see this we note that the notation can be chosen such that $v_{2}=z_{1}$ and $v_{3}$ is either $u_{2}$ (if (iii) holds) or $z_{2}$ (if (iv) holds). So if $z_{1}, v_{1}, u_{1}$ cannot play the role of $v_{1}, u_{1}, u_{2}$, then (iii) must hold and $u_{2}$ dominates $z_{1}$. But then $z_{1}, u_{1}, v_{1}$ can play the role of $v_{1}, u_{1}, u_{2}$.) So we can assume that (ii) does not hold. Hence (i) holds. Similarly, (iii) holds. So $v_{1}$ dominates and is dominated by both of $u_{1}, u_{2}$. By Lemma 3.1, there is no arc between $u_{1}$ and $u_{2}$. Let $y$ be the vertex $\neq v_{1}$ dominated by $u_{1}$. Then $u_{1}, y, v_{1}$ can play the role of $v_{1}, u_{1}, u_{2}$ in (3).

We are going to investigate the digraph $D_{1}$ defined in (3). The new vertex obtained by identifying $v_{1}$ and $u_{1}$ is denoted $u_{1}^{\prime}$. If the deletion of $v u_{1}$ and contradiction of $v u_{2}$ results in a digraph $D_{2}$ of minimum outdegree at least 2, then all statements below related to $D_{1}$ have counterparts related to $D_{2}$.

By Lemma 2.2, $D_{1}$ contains no weak 3-double-cycle. We claim that $D_{1}$ has at most three vertices of outdegree 2 . For when we contract $v_{1} u_{1}$ we "loose" a vertex of outdegree 2 . We may create a new vertex of outdegree 2 if $u_{1}$ dominates $v_{1}$ and $d^{+}\left(u_{1}, D\right)=3$, or if some vertex $w$ of outdegree 3 in $D$ dominates both $v_{1}$ and $u_{1}$. But only one such vertex $w$ can exist (by Lemma 3.2) and $w$ cannot exist if $u_{1}$ dominates $v_{1}$ (by Lemma 3.1). This proves the claim that $D_{1}$ has at most three vertices of outdegree 2. Therefore $D_{1}$ cannot be strongly 2 -connected. (If $D_{1}$ were strongly 2 -connected it would be a counterexample to Theorem 3.3 contradicting the minimality of $D$.) So $D_{1}$ contains a vertex $z_{1}$ such that $D_{1}-z_{1}$ is not strong. We choose $z_{1}$ such that $D_{1}-z_{1}$ is not strong, and we choose a terminal component $H_{1}$ of $D_{1}-z_{1}$ such that $H_{1}$ is relatively minimal. (That is, if $z^{\prime}$ is a vertex of $D_{1}$ and $H^{\prime}$ is a terminal component of $D_{1}-z^{\prime}$ such that $V\left(H^{\prime}\right) \subseteq V\left(H_{1}\right)$, then $H^{\prime}=H_{1}$.) Put $I_{1}=D_{1}-\left(V\left(H_{1}\right) \cup\left\{z_{1}\right\}\right)$.

(4) $u_{1}^{\prime} \in\left\{z_{1}\right\} \cup V\left(H_{1}\right)$ and $u_{2} \in V\left(I_{1}\right)$. 
Proof. If $u_{1}^{\prime} \in V\left(I_{1}\right)$, then $D-z_{1}$ would not be strong, contrary to (1). So $u_{1}^{\prime} \in\left\{z_{1}\right\} \cup V\left(H_{1}\right)$. If $u_{2} \notin V\left(I_{1}\right)$, then $D-z_{1}$ would fail to be strong (if $z_{1} \neq u_{1}^{\prime}$ ) and $D-u_{1}$ would fail to be strong (if $z_{1}=u_{1}^{\prime}$ ).

Now let $D_{1}^{\prime}$ be the $H_{1}$-reduction of $D_{1}$ at $z_{1}$. As all vertices of $D_{1}$ have outdegree $\geq 2, D_{1}^{\prime}$ has at least three vertices. Now we prove

(5) $D_{1}^{\prime}$ is strongly 2-connected.

Proof. We let $t$ denote any vertex of $D_{1}^{\prime}$ and show that $D_{1}^{\prime}-t$ is strong. This is clear if $t=z_{1}$ so assume $t \neq z_{1}$. The minimality of $H_{1}$ implies that each vertex of $D_{1}^{\prime}-t$ can reach $z_{1}$. So it only remains to show that, for each vertex $s$ in $D_{1}^{\prime}-t, D_{1}^{\prime}-t$ has a $z_{1}-s$ dipath. If $u_{1}^{\prime} \neq t$ and $u_{1}^{\prime} \neq z_{1}$, then we consider a dipath $P$ in $D-t$ from $z_{1}$ to $s$ (or to $v_{1}$ if $s=u_{1}^{\prime}$ ). If $P$ contains some arc leaving $I_{1}$, then we let $w_{1} w_{2}$ be the last such arc on $P$. The subdipath of $P$ from $w_{2}$ to $s$ contains no vertex of $I_{1}$. In particular, it does not contain the arc $v_{1} u_{2}$. Moreover, $z_{1}$ dominates $w_{2}$ in $D_{1}^{\prime}$. This shows that $D_{1}^{\prime}-t$ has a dipath from $z_{1}$ to $s$. If $u_{1}^{\prime}=z_{1}$ we argue similarly except that now $P$ denotes a $u_{1}-s$ dipath in $D-t$. If $u_{1}^{\prime}=t$ we also argue similarly except that now $P$ denotes a $z_{1}-s$ dipath in $D-u_{1}$.

As $D_{1}^{\prime}$ has fewer vertices than $D, D_{1}^{\prime}$ cannot be a counterexample to Theorem 3.3. By Lemma 2.3, $D_{1}^{\prime}$ contains no weak 3-double-cycles. Therefore, $D_{1}^{\prime}$ has at least four vertices of outdegree 2 . We now investigate the possibilities of where those four vertices can be. One possible vertex of outdegree 2 in $D_{1}^{\prime}$ is $z_{1}$. Other possibilities are $v_{2}$ and $v_{3}$. (Possibly $u_{1}=v_{2}$ or $v_{3}$.) Also there may be a vertex in $H_{1}$, that in $D$ has outdegree 3 and dominates both $v_{1}$ and $u_{1}$. Lemma 3.2 shows that there cannot be two such vertices. Finally, $u_{1}$ may have outdegree 3 in $D$ and dominate $v_{1}$. But if this is the case, then $D$ cannot have a vertex dominating both $v_{1}$ and $u_{1}$ by Lemma 3.1. As there are no other possibilities for vertices of outdegree 2 in $D_{1}^{\prime}$ we conclude

(6) $D_{1}^{\prime}$ has precisely four vertices of outdegree 2 . Three of them are $z_{1}, v_{2}, v_{3}$ (or $z_{1}, u_{1}^{\prime}, v_{i}$ in case $v_{5-i}=u_{1}$ where $i=2$ or 3 ). The fourth vertex of outdegree 2 is either $u_{1}^{\prime}$ (if $d^{+}\left(u_{1}, D\right)=3$ and $u_{1}$ dominates $v_{1}$ ) or a vertex that in $D$ has outdegree 3 and dominates both $v_{1}$ and $u_{1}$.

(6) shows that both of $v_{2}, v_{3}$ are in $H_{1}$ (except that one of them may equal $u_{1}$ ). It is possible that $u_{1}=v_{2}$ say. It is also possible that $z_{1}=u_{1}^{\prime}$. But both of these possibilities cannot occur since there are four vertices of outdegree 2 in $D_{1}^{\prime}$. By $(4), u_{2} \in V\left(I_{1}\right)$. So $d^{+}\left(u_{2}, D\right) \geq 3$ and none of $v_{2}, v_{3}$ dominates $u_{2}$. Hence the deletion of $v_{1} u_{1}$ and contraction of $v_{1} u_{2}$ results in a digraph $D_{2}$ of minimum outdegree at least 2 . We noted after (3) that the statements concerning $D_{1}$ have counterparts for $D_{2}$. The counterparts to statements (3), (4), (5), and (6) are denoted $\left(3^{\prime}\right),\left(4^{\prime}\right),\left(5^{\prime}\right)$, and $\left(6^{\prime}\right)$, respectively. As (6) implies that $d^{+}\left(u_{2}, D\right) \geq 3,\left(6^{\prime}\right)$ implies that $d^{+}\left(u_{1}, D\right) \geq 3$. In particular, $\left\{u_{1}, u_{2}\right\} \cap$ $\left\{v_{1}, v_{2}, v_{3}\right\}=\varnothing$ and $\left\{v_{2}, v_{3}\right\} \subseteq V\left(H_{1}\right) \backslash\left\{u_{1}^{\prime}\right\}$. After having proved (3) we 
introduced $D_{1}, z_{1}, H_{1}, I_{1}, D_{1}^{\prime}$. We define similarly $D_{2}, z_{2}, H_{2}, I_{2}, D_{2}^{\prime}$ (by interchanging $u_{1}$ and $\left.u_{2}\right)$. Now $\left(6^{\prime}\right)$ implies

(7) $I_{2}$ contains $u_{1}$ but not $u_{2}^{\prime} . H_{2}-u_{2}^{\prime}$ contains $v_{2}, v_{3}$.

$\left(6^{\prime}\right)$ also implies that either $u_{2}$ dominates $v_{1}$ in $D$ or else some vertex of outdegree 3 in $D$ dominates both $u_{2}$ and $v_{1}$. Such a vertex must be in $I_{1} \cup$ $\left\{z_{1}\right\}$. (Note that possibly $z_{1}=u_{1}^{\prime}$. But a vertex dominating $u_{2}$ and $v_{1}$ must be distinct from $u_{1}$ by Lemma 3.1.) Hence

(8) Some vertex of $I_{1} \cup\left\{z_{1}\right\}$ dominates $v_{1}$ in $D$.

(9) Either $z_{1} \neq u_{1}^{\prime}$ or $z_{2} \neq u_{2}^{\prime}$.

Proof. Suppose $z_{1}=u_{1}^{\prime}$ and $z_{2}=u_{2}^{\prime}$. The equality $z_{1}=u_{1}^{\prime}$ implies that every $v_{2}-u_{2}$ dipath in $D-v_{1}$ contains $u_{1}$. Similarly, the equality $z_{2}=u_{2}^{\prime}$ implies that every $v_{2}-u_{1}$ dipath in $D-v_{1}$ contains $u_{2}$. But $D-v_{1}$ contains a $v_{2}-\left\{u_{1}, u_{2}\right\}$ dipath. That dipath contradicts one of the two preceding statements.

By (9), the notation can be chosen such that $z_{1} \neq u_{1}^{\prime}$. Possibly $z_{2}=u_{2}^{\prime}$ and we can no longer interchange between $u_{1}$ and $u_{2}$. Below, therefore, we investigate $I_{2}$ and $H_{2}$.

First observe that, by $(8), z_{1}$ dominates $u_{1}^{\prime}$ in $D_{1}^{\prime}$. By (6) there is precisely one more vertex, say $r$, that in $D_{1}^{\prime}$ is dominated by $z_{1}$.

(10) If $z_{2}=u_{2}^{\prime}$ or $z_{2} \in V\left(I_{1}\right) \backslash\left\{u_{2}\right\}$, then $z_{1} \in V\left(H_{2}\right)$.

Proof. By (7), $v_{2} \in V\left(H_{2}\right)$. As $D$ is strongly 2-connected, $D-v_{1}$ has a $v_{2}-z_{1}$ dipath $P$. By the assumption of (10), $P$ does not contain $z_{2}$. Hence $P$ is in $\mathrm{H}_{2}$ (because $\mathrm{H}_{2}$ is a terminal component of $D_{2}-z_{2}$ and $P$ starts in that terminal component). In particular, the end $z_{1}$ of $P$ is in $H_{2}$.

(11) If $z_{2}=u_{2}^{\prime}$ or $z_{2} \in\left(V\left(H_{1}\right) \backslash\left\{u_{1}^{\prime}\right\}\right) \cup\left\{z_{1}\right\}$, then $I_{1}-u_{2} \subseteq H_{2}$.

Proof. Suppose first $z_{2}=u_{2}^{\prime}$. By (10), $z_{1} \in V\left(H_{2}\right)$. Every vertex in $I_{1}-u_{2}$ can be reached from $z_{1}$ in $D-u_{2}$. Any such dipath $P$ avoids $u_{1}, v_{1}$ and is in $D_{2}-z_{2}$. As $P$ starts in the terminal component $H_{2}$ of $D_{2}-z_{2}$ it also ends in $H_{2}$. Hence $I_{1}-u_{2} \subseteq H_{2}$.

Suppose next that $z_{2} \in\left(V\left(H_{1}\right) \backslash\left\{u_{1}^{\prime}\right\}\right) \cup\left\{z_{1}\right\}$. As $z_{2} \neq u_{2}^{\prime}$ we can apply $\left(6^{\prime}\right)$, which implies that $u_{2}^{\prime} \in V\left(H_{2}\right)$. As $D$ is strongly 2-connected, $D-z_{1}$ has dipaths from $u_{2}$ to all other vertices of $I_{1}$. As these dipaths are in $D_{2}-z_{2}$ (because $z_{2} \neq u_{2}^{\prime}$ ) and they start in the terminal component $\mathrm{H}_{2}$ of $\mathrm{D}_{2}-z_{2}$, we conclude that $I_{1}-u_{2} \in H_{2}$.

(12) If $z_{2} \in V\left(I_{1}\right) \backslash\left\{u_{2}\right\}$, then $\left(V\left(I_{1}\right) \backslash\left\{u_{2}, z_{2}\right\}\right) \cup\left\{z_{1}, u_{2}^{\prime}\right\} \subseteq V\left(H_{2}\right)$.

Proof. By (10), $z_{1} \in V\left(H_{2}\right)$. By $\left(6^{\prime}\right), u_{2}^{\prime} \in V\left(H_{2}\right)$. As $D-z_{2}$ has shortest dipaths from $\left\{z_{1}, u_{2}\right\}$ to all vertices of $I_{1}-\left\{u_{2}, z_{2}\right\}$ and these (shortest) dipaths are in $D_{2}-z_{2}$, we conclude that $I_{1}-\left\{u_{2}, z_{2}\right\} \subseteq H_{2}$.

By the definition of $\mathrm{H}_{2}$ and $\mathrm{I}_{2}$, there is no arc in $\mathrm{D}_{2}$ from $\mathrm{H}_{2}$ to $I_{2}$. As $u_{1} \in V\left(I_{2}\right)$ and $H_{2}$ contains almost all of $I_{1}$ (by (11) and (12)), there are not 
many arcs from $I_{1}$ to $u_{1}$. More precisely, (10), (11), and (12) imply

(13) There is at most one vertex (namely, one of $z_{1}, z_{2}, u_{2}$ ) in $I_{1} \cup\left\{z_{1}\right\}$ that in $D$ dominates $u_{1}$.

Proof. If $z_{2}=u_{2}^{\prime}$, we apply (10), (11). If $z_{2} \in V\left(I_{1}\right) \backslash\left\{u_{2}\right\}$ we apply (12). if $z_{2} \in\left(V\left(H_{1}\right) \backslash\left\{u_{1}^{\prime}\right\}\right) \cup\left\{z_{1}\right\}$ we apply (11). Note that if $z_{2}=z_{1}$, then $u_{2}^{\prime} \in V\left(H_{2}\right)$ and hence $u_{2}$ does not dominate $u_{1}$ in that case.

Let $G$ be the digraph obtained from the subdigraph of $D$ induced by $V\left(I_{1}\right) \cup$ $\left\{r, v_{1}, z_{1}\right\}$ by adding the arcs $r v_{1}, r z_{1}$ if they are not already present. As $D$ is strongly 2-connected $D$ contains, by Menger's Theorem, two $r-\left\{v_{1}, z_{1}\right\}$ dipaths that have only $r$ in common. These dipaths together with the subdigraph of $D$ induced by $V\left(I_{1}\right) \cup\left\{r, v_{1}, z_{1}\right\}$ is a subdivision of $G$. Hence $G$ contains no weak 3-double-cycle. Clearly, $d^{+}\left(v_{1}, G\right)=1$ and, therefore, we contract $v_{1} u_{2}$ into $u_{2}^{\prime}$ and denote the resulting digraph by $G^{\prime}$. By Lemma 2.2,

(14) $G^{\prime}$ contains no weak 3-double-cycle.

We shall prove that $G^{\prime}$ satisfies the assumption of Theorem 3.3 with $r$ playing the role of $v_{1}$.

(15) All vertices of $G^{\prime}$ have outdegree at least 2 in $G^{\prime}$.

Proof. Each vertex of $G$ (except $r$ and $v_{1}$ ) has the same outdegree in $G$ as in $D$ (where it has outdegree at least 3 ) unless it dominates $u_{1}$. So if a vertex has outdegree 1 in $G^{\prime}$, it would either have to dominate (in $D$ ) each of $v_{1}, u_{1}, u_{2}$ (which is impossible by Lemma 3.2), or it would have to equal $u_{2}^{\prime}$ (and $u_{2}$ would dominate both $v_{1}$ and $u_{1}$, which is impossible by Lemma 3.1), or it would equal $r$ (which clearly has outdegree 2 in $G$ and $G^{\prime}$ ).

(16) For any two vertices $z, z^{\prime}$ in $G^{\prime}-r, G^{\prime}-z^{\prime}$ has an $r-z$ dipath.

Proof. We can assume that $z \neq u_{2}^{\prime}, z_{1}$. As $D-z^{\prime}$ has an $r-z$ dipath (if $z^{\prime} \neq u_{2}^{\prime}$ ) and $D-u_{2}$ has an $r-z$ dipath, we easily get an $r-z$ dipath in $G^{\prime}-z^{\prime}$.

As $r$ can be reached from each vertex in $D-u_{1}$, we conclude that $r$ can be reached (in $G$ and hence also in $G^{\prime}$ ) from each vertex in $G^{\prime}$. (Note that any dipath in $D-u_{1}$ from $I_{1} \cup\left\{z_{1}\right\}$ to $r$ is in $G$ because $d^{+}\left(v_{1}, G\right)=1$.) Combining this with (16) we conclude that

(17) $G^{\prime}$ is strong.

We finally investigate the vertices of outdegree 2 in $G^{\prime}$. In $G^{\prime}, r$ has outdegree 2 . In $G$ all vertices (except $r, v_{1}$, and possibly one more) have outdegree $\geq 3$ by (13). When we form $G^{\prime}$ we may create a new vertex of outdegree 2 , namely, $u_{2}^{\prime}$ or a vertex dominating both $v_{1}$ and $u_{2}$. By Lemmas 3.1, 3.2 only one new vertex of outdegree 2 is created in this way. So

(18) $G^{\prime}$ has at most three vertices of outdegree 2 .

(14)-(18) imply that $G^{\prime}$ is a counterexample to Theorem 3.3. This contradiction to the minimality of $D$ completes the proof. 


\section{EVEN DIGRAPHS}

Theorem 3.3 immediately implies

Theorem 4.1. If all vertices (except possibly three) in a strongly 2-connected digraph $D$ have outdegree at least 3 , then $D$ is even.

We shall show that Theorem 4.1 is best possible in a strong sense.

The 4-double-cycle is strongly 2-connected and is not even. It has 4 vertices of outdegree 2. Another such example is the digraph $D_{1}$ consisting of a dicycle $x_{1} x_{2} x_{3} x_{4} x_{5} x_{1}$ and the additional arcs $x_{2} x_{4}, x_{2} x_{5}, x_{5} x_{2}, x_{4} x_{1}, x_{3} x_{1}, x_{1} x_{3}$.

$D_{1}$ is strongly 2 -connected and has precisely four vertices of outdegree 2 . It contains no weak odd double-cycle and therefore is not even.

In Theorem 4.1 it is also important that $D$ is strongly 2-connected. Indeed, there are infinitely many strong digraphs of minimum indegree 2 and minimum outdegree 3 that are not even. We give here just one example. Let $D_{2}$ be obtained from $D_{1}$ above by adding a new vertex $y$ such that $y$ dominates $x_{1}$ and is dominated by $x_{1}, x_{3}, x_{4}, x_{5}$. Then $d^{+}\left(y, D_{2}\right)=1$ and, if we contract $y x_{1}$, then we obtain $D_{1}$. As $D_{1}$ is not even it follows by Theorem 2.1 and Lemma 2.2 that $D_{2}$ is not even. Now take three disjoint copies of $D_{2}$ and identify the three $y$-vertices into one vertex. Then the resulting digraph is strong, noneven, and has minimum in- and outdegree 2 and 3, respectively. However, if the minimum in- and outdegree are both at least 3 , the situation changes.

Theorem 4.2. If $D$ is a strong digraph of minimum in- and outdegree at least 3 , then $D$ is even.

Proof. If $D$ is strongly 2-connected we apply Theorem 4.1. So assume $D$ has a vertex $v$ such that $D-v$ is not strong. Let $H$ be either an initial or terminal component of $D-v$. We chose $v$ and $H$ such that $|V(H)|$ is minimum. Suppose $H$ is a terminal component. Let $D^{\prime}$ be the $H$-reduction of $D$ at $v$. We claim that $D^{\prime}$ is strongly 2 -connected. So we let $v^{\prime}$ denote any vertex of $D^{\prime}$ and we shall prove that $D^{\prime}-v^{\prime}$ is strong. This is clear if $v^{\prime}=v$. So assume that $v^{\prime} \neq v$. If $D^{\prime}-v^{\prime}$ is not strong we let $H^{\prime}$ be a terminal or initial component of $D^{\prime}-v^{\prime}$ not containing $v$. But then $H^{\prime}$ is also a terminal or initial component of $D-v^{\prime}$ contradicting the minimality of $H$. So $D^{\prime}$ is strongly 2-connected. Also $D^{\prime}$ has at most one vertex of outdegree $<3$, namely, $v$.

By Theorem 4.1, $D^{\prime}$ has a weak 3-double-cycle. By Lemma 2.3, $D$ has a weak 3-double-cycle. Hence $D$ is even.

Corollary 4.3. Every strongly 3-connected digraph contains a dicycle of even length.

In [14] it is pointed out that there exists a strongly 2-connected digraph $D^{7}$ on 7 vertices that has no dicycle of even length (namely, the one that is the union of the two dicycles $x_{1} x_{2} x_{3} x_{4} x_{5} x_{6} x_{7} x_{1}$ and $\left.x_{1} x_{4} x_{7} x_{3} x_{6} x_{2} x_{5} x_{1}\right)$. It was asked if there are infinitely many such digraphs. This is still open. However, we get the following weaker statement.

Proposition 4.4. There are infinitely many strongly 2-connected digraphs that are not even. 
Proof. Let $G$ be a graph drawn in the Euclidean plane such that all edges are straight line segments and such that no two edges cross. (Graph and edge are defined in the next section.) Let $p$ be a point not on $G$ such that no half line starting at $p$ contains an edge of $G$. Orient every edge in the clockwise direction around $p$. The resulting digraph $D$ is not even. (Fix a half line $L$ starting at $p$ and assign weight 1 to an arc of $D$ if and only if the arc intersects $L$.) It is easy to describe $G$ and $p$ such that $D$ is strongly 2-connected.

A digraph is $k$-diregular if all vertices have indegree and outdegree $k$. Friedland [2] proved that every $k$-diregular digraph is even for $k \geq 7$. He conjectured that this also holds for $k \geq 3$. This conjecture follows from Theorem 4.2 since every terminal component of a $k$-diregular digraph is $k$-diregular. Vazirani and Yannakakis [17] pointed out that a proof of Lovász's conjecture would follow from [2] if one could prove that, for $k$ sufficiently large, every strongly $k$-connected digraph contains a 7-diregular subgraph. If so, it would also contain a 3-diregular subgraph (since every $m$-diregular digraph is the union of $m$ 1-diregular subgraphs). But this last statement is incorrect. By a result of Pyber and Szemeredi (see [9]) there exists, for each $k$, a graph $G$ such that all vertices have degree $\geq k$ and $G$ has no 3-regular subgraph. (Graph and degree are defined in the next section. 3-regular means that all vertices have degree 3.) It is no loss of generality to assume that $G$ is bipartite (as every graph of minimum degree $2 k-1$ contains a bipartite graph of minimum degree $k$, see $[9,15]$. Let $V_{1}, V_{2}$ be the bipartition of $V(G)$. Let $G^{\prime}$ be another copy of $G$ where $V_{1}^{\prime}, V_{2}^{\prime}$ correspond to $V_{1}$ and $V_{2}$, respectively. Now form the disjoint union $G \cup G^{\prime}$. Direct all edges from $V_{1} \cup V_{2}^{\prime}$ to $V_{2} \cup V_{1}^{\prime}$. Add all arcs from $V_{2} \cup V_{1}^{\prime}$ to $V_{1} \cup V_{2}^{\prime}$. The resulting digraph is strongly $k$-connected and has no 3-diregular subgraph.

\section{APPLICATIONS TO COLOURINGS OF HYPERGRAPHS, SIGN-NONSINGULAR MATRICES, AND PERMANENTS}

In this section we apply Theorem 4.2 to the problems mentioned in the introduction.

A hypergraph $H$ is a pair $V, E$ where $V$ is a finite set of vertices and $E$ is a collection of subsets called hyperedges of $V$ each of cardinality at least 2 . The number of hyperedges containing the vertex $v$ is the degree of $v$. If all hyperedges have cardinality 2 they are called edges and the hypergraph is called a graph. The hypergraph $H$ is bipartite if there exists a partition $V=V_{1} \cup V_{2}$ such that each hyperedge intersects both $V_{1}$ and $V_{2} . H$ is minimally nonbipartite if $H$ is not bipartite but every proper subhypergraph is bipartite. Now let $D$ be a digraph on $n$ vertices. We now define a hypergraph $H_{D}$ as follows. The vertex set of $H_{D}$ is $V(D)$. For every vertex $v$ in $D$ we let $E_{v}$ consist of $v$ and the vertices dominated by $v$. Now the hyperedge set of $H_{D}$ is the collection of the sets $E_{v}, v \in V(D)$. Seymour [11] proved that $H_{D}$ is minimally nonbipartite if and only if $D$ is strong and has no dicycle of even length. He also proved that every minimally nonbipartite hypergraph $(V, E)$ with $|V|=|E|$ is of the form $H_{D}$. From the results in [13] it follows that the cardinalities of all hyperedges in such a hypergraph may be greater than any (fixed) natural number. But 
Theorem 4.2 implies

Theorem 5.1. If $H=(V, E)$ is a minimally nonbipartite hypergraph with $|V|=$ $|E|$, then either some hyperedge of $H$ has cardinality $\leq 3$ or some vertex has degree $\leq 3$.

An $n$ by $n$ real matrix $A=\left[a_{i j}\right]$ is sign-nonsingular if $A$ is nonsingular and all nonzero terms in the standard expression of the determinant $\operatorname{det} A$ have the same sign. By permuting rows and multiplying some rows by -1 , if necessary, we may assume that all entries $a_{i i}$ in the main diagonal are positive. Now we form a digraph $D$ with vertex set $\left\{v_{1}, v_{2}, \ldots, v_{n}\right\}$ such that $v_{i}$ dominates $v_{j}$ if $a_{i j} \neq 0$. If $a_{i j}>0$ (respectively $a_{i j}<0$ ) we assign the weight 1 (respectively 0 ) to the arc $v_{i} v_{j}$. Now one can easily show (see [4]) that $A$ is sign-nonsingular if and only if $D_{A}$ has no dicycle of even total weight. Let us say that an entry $a_{i j}$ is redundant if $a_{i j} \neq 0$ but $a_{i j}$ is not a factor of any nonzero term in the standard expression of $\operatorname{det} A$ (or, equivalently, the arc $v_{i} v_{j}$ is in no dicycle of $\left.D_{A}\right)$.

By the results of [13] there are sign-nonsingular matrices whose rows all have many nonzero entries. But Theorem 4.2 implies

Theorem 5.2. If $A$ is a sign-nonsingular square matrix with no redundant entries, then some row or some column of $A$ has at most 3 nonzero entries.

We now turn to Polya's problem [8]. For simplicity we consider an $n$ by $n$ 0-1 matrix $A=\left[a_{i j}\right]$ with $1-s$ in the main diagonal. A modification of $A$ is a matrix $A^{\prime}$ obtained from $A$ by replacing some $1-s$ by -1 . We say that $A^{\prime}$ is a good modification if $\operatorname{per} A=\operatorname{det} A^{\prime}$. We let $G_{A}$ be the bipartite graph with vertex set $V_{1} \cup V_{2}$ when $V_{1}=\left\{r_{1}, r_{2}, \ldots, r_{n}\right\}, V_{2}=\left\{c_{1}, c_{2}, \ldots, c_{n}\right\}$ and $G_{A}$ has the edge $\left\{r_{i}, c_{j}\right\}$ if $a_{i j}=1$. Then per $A$ is the number of perfect matchings (i.e., subgraphs in which all vertices have degree 1 ) in $G_{A}$. A Pfaffian orientation of a graph $G$ is an assignment of orientations to every edge such that the resulting digraph $D$ has the following property: If $M_{1}, M_{2}$ are two perfect matchings in $G$, then each cycle in $M_{1} \cup M_{2}$ has an odd number of edges oriented in both directions (of the cycle). Now the following three statements below are equivalent:

(i) $A$ has a good modification.

(ii) $G_{A}$ has a Pfaffian orientation.

(iii) The digraph $D_{A}^{\prime}$ obtained from $G$ by directing the edges $\left\{r_{i}, c_{i}\right\}$ from $V_{1}$ to $V_{2}$ and all other edges from $V_{2}$ to $V_{1}$ is not even.

The equivalence of (i) and (ii) is due to Kasteleyn [3], and the equivalence of (ii) and (iii) is due to Vazirani and Yannakakis [17].

Note that, by Lemma 2.2, the digraph $D_{A}^{\prime}$ is even iff the digraph $D_{A}^{\prime \prime}$ obtained by contracting all arcs $r_{i} c_{i}(i=1,2, \ldots, n)$ is even. Thus Theorem 4.2 implies

Theorem 5.3. If $A$ is a nonnegative real $n$ by $n$ matrix and $A$ has no redundant entry and each row and column has at least 4 positive elements, then $A$ has no good modification. 
Theorem 5.4. If $G$ is a bipartite graph of minimum degree at least 4 such that each edge is contained in a perfect matching, then $G$ has no Pfaffian orientation.

Little [5] showed that a graph has a Pfaffian orientation if it contains no $K_{3,3^{-}}$ subdivision. $\left(K_{3,3}\right.$ is the graph with six vertices $v_{1}, v_{2}, v_{3}, u_{1}, u_{2}, u_{3}$ and nine edges $\left\{v_{i}, u_{j}\right\}, i, j=1,2,3$.) Using Theorem 2.1 and the equivalence of (ii) and (iii) above, one can prove the following:

Theorem 5.5. Let $G$ be a bipartite graph with bipartition $V(G)=V_{1} \cup V_{2}$. Then $G$ has no Pfaffian orientation if and only if $G$ contains a subgraph $H$ such that $H$ is a $K_{3,3}$-subdivision with $\left\{v_{1}, v_{2}, v_{3}\right\} \subseteq V_{1}$ and $\left\{u_{1}, u_{2}, u_{3}\right\} \subseteq V_{2}$ and $G-V(H)$ has a perfect matching.

It would be interesting to extend Theorem 5.5 to nonbipartite graphs. The Petersen Graph shows that this is not immediately possible. But the Petersen graph might somehow be the only obstacle as it is the matching theorem of Lovász in [7].

Another interesting problem is to decide if there exists a polynomial time algorithm for deciding if a given digraph has an even length dicycle. For planar digraphs a polynomial time algorithm is described in [16].

\section{REFERENCES}

1. N. Alon and N. Lineal, Cycles of length $O$ modulo $k$ in directed graphs, J. Combin. Theory Ser. B 47 (1989), 114-119.

2. S. Friedland, Every 7-regular digraph contains an even cycle, J. Combin. Theory Ser. B $\mathbf{4 6}$ (1989), 249-252.

3. P. W. Kasteleyn, Graph theory and crystal physics, Graph Theory and Theoretical Physics (F. Harary, ed.), Academic Press, New York, 1967, pp. 43-110.

4. V. Klee, R. Ladner, and R. Manber, Sign-solvability revisited, Linear Algebra Appl. 59 (1984), 131-158.

5. C. H. C. Little, An extension of Kasteleyn's method of enumerating the 1-factors of planar graphs, Combinatorial Mathematics, Proceedings 2nd Australian Conference (D. Holton, ed.), Lecture Notes in Math., vol. 403, Springer, Berlin, 1974, pp. 63-72.

6. L. Lovász, Problem 2, Recent Advances in Graph Theory (M. Friedler, ed.), Proceedings Symposium Prague, 1974, Academia Praha, Prague, 1975.

7. __ Matching structure and the matching lattice, J. Combin. Theory Ser. B 43 (1987), 187-222.

8. G. Polya, Aufgabe 424, Arch. Math. Phys. Ser. (3) 20 (1913), 271.

9. L. Pyber, Regular subgraphs of dense graphs, Combinatorica 5 (1985), 347-349.

10. H. Sachs, Perfect matchings in hexagonal systems, Combinatorica 4 (1984), 89-99.

11. P. D. Seymour, On the two coloring of hypergraphs, Quart. J. Math. Oxford Ser. (3) 25 (1974), 303-312.

12. P. Seymour and C. Thomassen, Characterization of even directed graphs, J. Combin. Theory Ser. B 42 (1987), 36-45.

13. C. Thomassen, Even cycles in directed graphs, European J. Combin. 6 (1985), 85-89.

14. _ Sign-nonsingular matrices and even cycles in directed graphs, Linear Algebra Appl. 75 (1986), 27-41.

15. _ Paths, circuits and subdivisions, Selected Topics in Graph Theory (L. W. Beineke and R. L. Wilson, eds.), vol. 3, Academic Press, 1988, pp. 97-131. 
16.

17. V. V. Vazirani and M. Yannakakis, Pfaffian orientations, $0-1$ permanents, and even cycles in directed graphs, Discrete Appl. Math. 25 (1989), 179-190.

ABSTRACT. If each arc in a strongly connected directed graph of minimum indegree and outdegree at least 3 is assigned a weight 0 or 1 , then the resulting weighted directed graph has a directed cycle of even total weight. This proves a conjecture made by L. Lovász in 1975 and has applications to colour-critical hypergraphs, sign-nonsingular matrices, and permanents of matrices.

Mathematical Institute, Technical University of Denmark, DK-2800 Lyngby, DenMark 